\title{
Optimizing patient outcomes by improving STEMI target times
}

\section{Danielle Binda, Team Members: SHC ED STEMI Project Team}

Background: It is widely recognized that delays in STEMI identification and treatment can negatively affect patient outcomes. Early intervention to achieve reperfusion of the blocked vessel is crucial in minimizing myocardial damage and optimizing patient outcomes. In April 2017, the Southern Alberta STEMI program identified a delay in SHC ED achieving STEMI target times as outlined by the American College of Cardiology and the American Heart Association.

Of all walk-in STEMI patients presenting to SHC ED between October 2016 and October 2017 requiring urgent transfer to FMC cardiac cath lab:

- $24 \%$ met the 'Triage to $1^{\text {st }}$ ECG target' of $\leq 10$ mins

- $6 \%$ met the 'Door in-Door out target' of $\leq 30$ mins

A multi-disciplinary project team was formed to examine barriers to both targets and begin implementing strategies aimed at improving these times.

Implementation: The AHS AIW framework as well as the IHI Model for Improvement were useful in guiding our work. A clearly defined problem statement and project goal assisted the project team to focus on engagement of key stakeholders. A multi-disciplinary project team was formed that included ED physician, ED CNE, ED RNs, unit clerks, SHC cardiotech staff and manager, EMS deployment manager who met approx. q2 months. Brainstorming sessions were completed to identify delays affecting each target and intervention strategies were developed to directly impact each identified delay. The project was implemented in 2 phases.

Phase 1 rolled out in July 2018 with interventions aimed at improving the Triage to ECG target (stretcher for ECGs at triage, cardiotech prioritizing r/o STEMI ECGs, “STEMI notification” call out to ED code team, etc) . Regular feedback was provided to ED staff via email and posters to increase buy in for change adoption.

Phase 2 rolled out in October 2018 with interventions aimed at improving the Door in-Door out (DIDO) target (formalized 'Hot Swap' process with EMS crew in EMS park as primary transfer to cath lab, streamlined booking process for Red/STAT transfers to cath lab, RN accompanying pt. in ambulance, transfer checklist, INFO sessions post STEMI transfer).

Sustainability planning includes standardizing ECGs at triage for symptoms of cardiac ischemia, including process education into RN orientation, development of STEMI workbook in tableau for ongoing monitoring and regular STEMI SIMs. Project wrap up completed Sept 2019 with plan for team to meet again in 6 months to address any process gaps.

Evaluation Methods: STEMI is not a high-volume pt. presentation to the ED (43 walk-in STEMI pts in 1 year at SHC ED) so PDSA cycles were a challenge. STEMI patient data was provided to the project team by the STEMI Program data coordinator every 3 months for chart review. Initial review was more thorough to aid in identification of possible opportunities for improvement (med administration, extensive diagnostics prior to cath lab transfer, etc). Triage to $1^{\text {st }}$ ECG and DIDO times were calculated, monitored over time and reviewed with the project 
team at each meeting. The "Weekly ECG acquisition" tableau workbook was used to monitor Triage to ECG times for all patients presenting with cardiac chest pain. An unintended consequence of placing an ECG stretcher at triage developed as triage RNs began completing ECGs on more patients than intended (ie; vertigo, bradycardia, a-fib) reducing availability for patients presenting with cardiac ischemia.

Results: Between July 2018-July 2019, 43 walk-in STEMI patients presented to SHC ED and were transferred to the FMC cath lab for revascularization.

Target \#1: Triage to $1^{\text {st }} \mathrm{ECG} \leq 10 \mathrm{mins}$

- $50 \%$ reduction in median time for Triage to $1^{\text {st }}$ ECG time

- $51 \%$ increase in target being met

Target \#2: Door in-Door out (DIDO) $\leq 30$ mins

- $44 \%$ reduction in median time for DIDO time

- $28 \%$ increase in target being met

Triage to physician assessment time was also reduced by $40 \%$ and EMS Transport booked to ED arrival time also reduced by $33 \%$

\section{Advice and Lessons Learned:}

1) Develop a clear problem and goal/aim statement. Use baseline data to validate the problem and ensure the team has a mechanism to regularly provide results to staff.

2) Low \# of STEMI presentations made rapid tests of change a challenge. Had to collect data retrospectively every 3 months to review and make changes. We eventually developed a feedback form to aid in more rapid data collection $\&$ analysis, but this was not reliably completed by bedside staff

3) Involving key stakeholders is critical. We involved members of the cardiotech team at each meeting to ensure that strategies we were putting into place weren't creating downstream workflow issues for them. This increased buy in for the cardiotechs to complete more timely ECGs and involved them in the solution. 